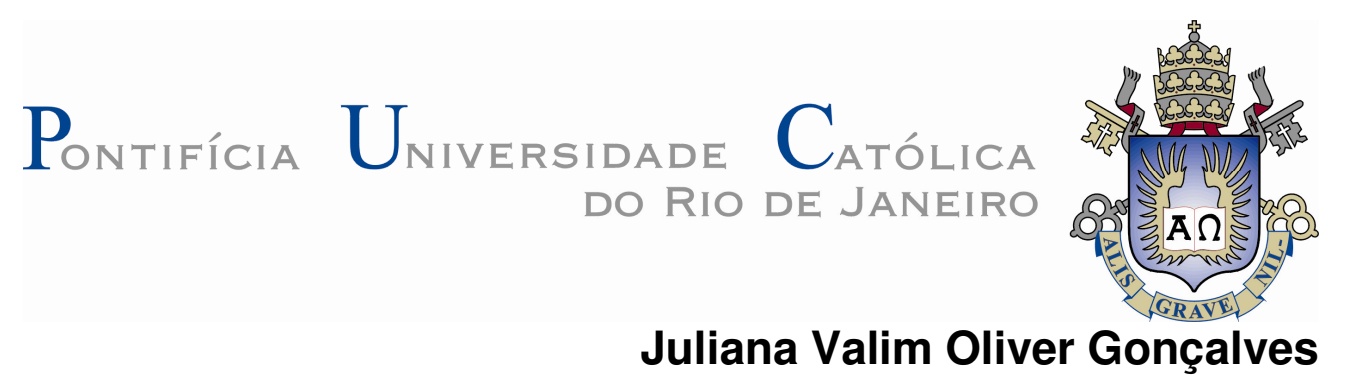

\author{
Variabilidade do Sinal, Banda de Coerência e \\ Espalhamento Temporal em Ambiente de Rádio \\ Propagação Móvel em 3,5GHz
}

\begin{abstract}
Dissertação de Mestrado
Dissertação apresentada como requisito parcial para obtenção do título de Mestre pelo Programa de PósGraduação em Engenharia Elétrica da PUC-Rio.
\end{abstract}

Orientador: Prof. Gláucio Lima Siqueira 


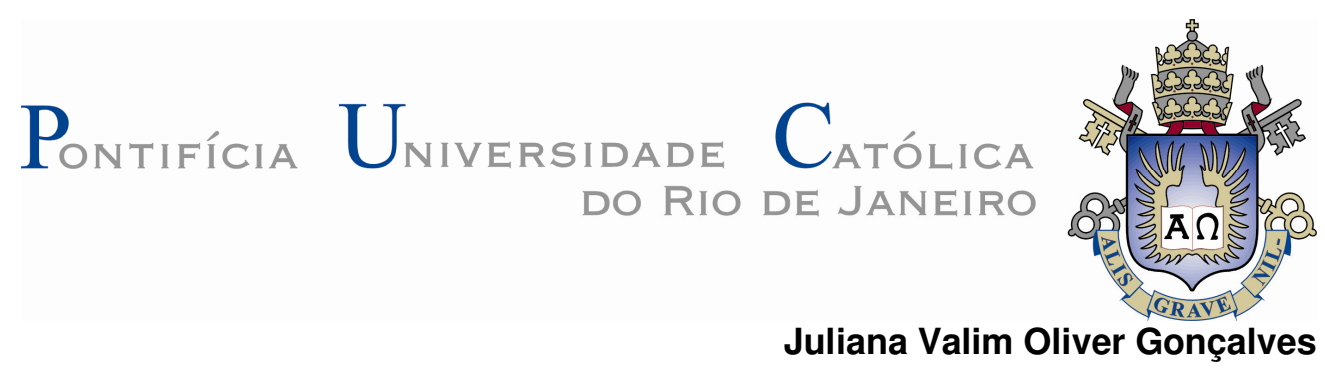

\section{Variabilidade do Sinal, Banda de Coerência e Espalhamento Temporal em Ambiente de Rádio Propagação Móvel em 3,5GHz}

Dissertação apresentada como requisito parcial para obtenção do título de Mestre pelo Programa de Pós-Graduação em Engenharia Elétrica da PUC-Rio, aprovada pela Comissão Examinadora abaixo assinada.

Dr. Gláucio Lima Siqueira Orientador Centro de Estudos em Telecomunicações - PUC-Rio

Dr. Luiz Alencar Reis da Silva Mello Centro de Estudos em Telecomunicações - PUC-Rio

Dr. Eduardo Javier Arancibia Vásquez CLARO RJ/ES

José Eugênio Leal Coordenador(a) Setorial do Centro Técnico Científico - PUC-Rio 
Todos os direitos reservados. É proibida a reprodução total ou parcial do trabalho sem autorização da universidade, da autora e do orientador.

Juliana Valim Oliver Gonçalves

Graduou-se em Engenharia de Telecomunicações, no Instituto de Estudos Superiores da Amazônia - IESAM, em Belém/PA, em 2006. Em 2007 ingressou no Programa de Pós-graduação em Engenharia Elétrica - Mestrado na área de eletromagnetismo aplicado no Centro de Estudos em Telecomunicações da Pontifícia Universidade Católica do Rio de Janeiro.

Ficha Catalográfica

Valim Gonçalves, Juliana Oliver

Variabilidade do sinal, banda de coerência e espalhamento temporal em ambiente de rádio propagação móvel em 3,5GHz / Juliana Valim Oliver Gonçalves ; orientador: Gláucio Lima Siqueira. - 2009.

143 f. : il. (color.) ; $30 \mathrm{~cm}$

Dissertação (Mestrado em Engenharia Elétrica)Pontifícia Universidade Católica do Rio de Janeiro, Rio de Janeiro, 2009.

Inclui bibliografia

1. Engenharia elétrica - Teses. 2. Canal de rádio propagação móvel. 3. Desvanecimento em pequena e larga escala. 4. Banda de coerência do canal. 5. Espalhamento de retardos (delay spread). I. Siqueira, Gláucio Lima. II. Pontifícia Universidade Católica do Rio de Janeiro. Departamento de Engenharia Elétrica. III. Título.

CDD: 621.3 
Àqueles que estiveram ao meu lado e me apoiaram À minha família e amigos por estarem sempre presentes. 


\section{Agradecimentos}

Ao professor orientador, Dr. Gláucio Lima Siqueira, pelas sábias palavras de orientação durante todo o percurso da pesquisa e elaboração desta dissertação.

Ao INMETRO pela disponibilização dos equipamentos de medição.

À PUC-Rio pela bolsa de isenção proporcionada e a CAPES pela bolsa de fomento fornecida.

Ao Carlos Rodriguez pela parceria ao longo das medições, discussões intermináveis e disponibilização da viatura de medição.

Ao Dr. Pedro Vladimir Gonzalez Castellanos e Davi Guedes pela cooperação na elaboração dos arquivos em MatLab.

Ao Prof. Dr. Marco Antonio Grivet pelos seus valiosos esclarecimentos.

À Prof. Dra. Terezinha Valim O. Gonçalves pelas sugestões de composição deste texto. 


\section{Resumo}

Valim Gonçalves, Juliana Oliver; Siqueira, Gláucio Lima. Variabilidade do Sinal, Banda de Coerência e Espalhamento Temporal em Ambiente de Rádio Propagação Móvel em 3,5GHz. Rio de Janeiro, 2009. 143p. Dissertação de Mestrado - Departamento de Engenharia Elétrica, Pontifícia Universidade Católica do Rio de Janeiro.

Nesta dissertação, é apresentado estudo realizado sobre a variabilidade do sinal, da banda de coerência e do espalhamento de retardos em um ambiente de rádio propagação móvel, por meio de medição em campo de um sinal WiMAX propagado ao longo do canal rádio móvel. A análise da estatística do sinal capturado é comparada com valores teóricos. O sinal WiMAX utilizado possui a tecnologia OFDM-256, como base da sua camada física, e frequência central de 3,410 GHz. A partir dos dados coletados durante as medições, são realizadas as análises da variabilidade do sinal, taxa de cruzamento de nível, tempo médio de desvanecimento, verificada a banda de coerência do canal e calculado o espalhamento de retardos (delay spread), por meio de fórmulas teóricas, em ambientes de LOS e NLOS. O nível do sinal medido é apresentado na forma de distribuição de probabilidade cumulativa, comparado com as distribuições cumulativas teóricas Rayleigh e m-Nakagami, sendo possível verificar a satisfatória adaptação dos dados às distribuições teóricas. São apresentados gráficos com a informação de taxa de cruzamento de nível e tempo médio de desvanecimento de determinadas subportadoras do sinal capturado, bem como os valores teóricos dos parâmetros supracitados, de acordo com as distribuições Rayleigh e m-Nakagami. Novamente, é possível verificar a satisfatória adaptação entre as informações teóricas e os dados medidos em campo. Por fim, é realizada a correlação entre as subportadoras, com o intuito de averiguar a banda de coerência definida, neste trabalho, para um valor de correlação igual a 0,5. De posse dessa informação, o cálculo do parâmetro de espalhamento de retardos é realizado, com base em fórmulas presentes na literatura.

\section{Palavras-chave}

Canal de rádio propagação móvel; desvanecimento em pequena e larga escala; banda de coerência do canal; espalhamento de retardos (delay spread). 


\section{Abstract}

Valim Gonçalves, Juliana Oliver; Siqueira, Gláucio Lima (Advisor). Signal Variability, Coherence Bandwidth and Delay spread on Mobile Radio Propagation Environment at 3.5GHz. Rio de Janeiro, 2009. 143p. MSc Dissertation - Departamento de Engenharia Elétrica, Pontifícia Universidade Católica do Rio de Janeiro.

This work presents a study of signal variability, coherence bandwidth and delay spread of a WiMAX signal transmitted through a mobile radio propagation environment. The statistical analysis of the received signal is compared with theoretical distributions. The transmitted WiMAX signal has its physical layer based on OFDM-256 and $3.4 \mathrm{GHz}$ as central frequency. Based on the signal received during the measurements campaign some analysis are done: signal variability; level crossing rate; average fade duration; coherence bandwidth; and delay spread calculation, based on theoretical formulas, in LOS and NLOS environment. The received signal amplitude is plotted as a function of the cumulative probability and compared to the theoretical Rayleigh and $\mathrm{m}-\mathrm{Nakagami}$ cumulative distributions. It's possible to notice a good characterization of the measured data based on those two distributions. Some graphs show the level crossing rate and average fade duration of a specific subcarrier of the received signal. In the same graphs are also plotted the theoretical values of Nakagami-m and Rayleigh distributions for those two parameters and we are able to see the good agreement. The correlation between subcarriers is also calculated in order to find out the correlation bandwidth for a correlation of 0.5 . Once that the correlation bandwidth is known, the delay spread is calculated based on theoretical formulas.

\section{Keywords}

Mobile radio propagation channel; small scale fading; coherence bandwidth; delay spread. 


\section{Sumário}

$\begin{array}{ll}\text { Introdução } & 15\end{array}$

$\begin{array}{lr}\text { 1. Tecnologia WiMAX } & 18\end{array}$

1.1. Padrão WiMAX 20

1.1.1. Camada Física (PHY) 21

1.2. Camada MAC 25

1.3. Aspectos sistêmicos 26

1.3.1. Multiplexação por Divisão de Frequências Ortogonais - OFDM 26

1.3.2. SubCanalização em WiMAX 29

$\begin{array}{ll}\text { 1.3.3. Modulação adaptativa } & 31\end{array}$

$\begin{array}{ll}\text { 1.3.4. Arquitetura de rede } & 31\end{array}$

1.4. Tecnologias competitivas 32

1.5. OFDM - Multiplexação por Divisão de Frequências Ortogonais 33

2. Canal de rádio propagação móvel 38

2.1. Atenuação com a distância, desvanecimento em pequena e larga $\begin{array}{ll}\text { escala } & 40\end{array}$

2.2. Mecanismos de propagação 44

2.3. Características do canal de rádio propagação móvel 45

2.3.1. Perfil de retardos (Power delay profile) 46

2.3.2. Banda de coerência $\quad 47$

2.3.3. Espalhamento Doppler 48

$\begin{array}{ll}\text { 2.3.4. Tempo de coerência } & 49\end{array}$

3 . Análise estatística do sinal 51

3.1. Distribuição log-Normal 52

3.2. Distribuição Rayleigh

3.2.1. Taxa de cruzamento de nível e duração média do desvanecimento 57

3.3. Distribuição de Rice $\quad 58$

3.3.1. Taxa de cruzamento de nível e duração média do desvanecimento 60

3.4. Distribuição m-Nakagami 60

3.4.1. Taxa de cruzamento de nível e duração média do desvanecimento 62 
4. Campanhas de medidas e equipamentos de medição 65

4.1. Sistema de medidas 66

4.2. Primeira campanha de medição: varredura de espectro 71

4.3. Segunda campanha de medição: determinação do raio de cobertura da antena transmissora

4.4. Terceira campanha de medição: aquisição da informação de fase e quadratura do sinal transmitido para realização das análises estatísticas

4.4.1. Características do sinal, parâmetros de transmissão e recepção $\quad 74$

4.4.2. Balanço de potência $\quad 76$

$\begin{array}{ll}\text { 4.4.3. Frequência de amostragem } & 78\end{array}$

5. Resultados $\quad 79$

5.1. Variabilidade do sinal $\quad 79$

5.1.1. Função distribuição cumulativa $\quad 80$

5.1.2. Desvanecimentos em ambientes com e sem linha de visada 88

5.1.3. Taxa de cruzamento de nível e duração média do desvanecimento 90

5.2. Banda de coerência 107

5.3. Espalhamento temporal (Delay Spread) 117

6. Conclusões 119

6.1. Estatística do sinal 119

6.2. Banda de coerência $\quad 121$

6.3. Espalhamento de retardos (Delay Spread) 123

6.4. Trabalhos futuros 123

$\begin{array}{ll}\text { Referências } & 125\end{array}$

$\begin{array}{ll}\text { Apêndices } & 130\end{array}$

$\begin{array}{ll}\text { Apêndice A } & 131\end{array}$

$\begin{array}{ll}\text { Apêndice } B & 136\end{array}$

$\begin{array}{ll}\text { Apêndice C } & 138\end{array}$ 


\section{Lista de figuras}

Figura 1.1: Multiplexações FDM e OFDM: (a) espectro FDM;

(b) espectro OFDM.

Figura 1.2: Sinal recebido com transmissão de portadora única e OFDM. $\quad 27$

Figura 1.3: Estrutura do símbolo OFDM no domínio do tempo. 28

Figura 1.4: Subportadoras OFDM no domínio da frequência. 28

Figura 1.5: Subportadoras OFDMA no domínio da frequência. 29

Figura 1.6: Subcanalização do sistema WiMAX: (a) OFDM; (b) OFDMA. 30

Figura 1.7: Modulação adaptativa. $\quad 31$

Figura 1.8: Arquitetura WiMAX 802.16-2004. 32

Figura 1.9: Cobertura WiFi e WiMAX. 33

Figura 1.10: Subportadoras de um sinal OFDM: (a) domínio da frequência; (b) domínio do tempo. 35

Figura 1.11: Diagrama de blocos de um modulador OFDM. 37

Figura 2.1: Componentes em fase e quadratura em coordenada polar. $\quad 39$

Figura 2.2: Componentes em fase e quadratura em coordenadas cartesiana e polar. 39

Figura 2.3. Típico sinal recebido por uma unidade móvel. 40

Figura 2.4: Desvanecimento em larga escala. $\quad 42$

Figura 2.5: Desvanecimento em pequena escala. 43

Figura 2.6: Reflexão. $\quad 44$

Figura 2.7: Difração. $\quad 45$

Figura 2.8: Perfil de retardos. $\quad 46$

Figura 2.9: Efeito Doppler. $\quad 49$

Figura 3.1: Gráfico da função densidade de probabilidade log-Normal. 53

Figura 3.2: Gráfico da função densidade de probabilidade Gaussiana. 53

Figura 3.3: Gráfico da função de distribuição cumulativa: (a) lognormal; (b) normal. $\quad 54$

Figura 3.4: Função densidade de probabilidade Rayleigh. 55

Figura 3.5: Função distribuição cumulativa Rayleigh. 57

Figura 3.6: Gráfico da função densidade de probabilidade de Rice: (a)

$K \rightarrow 0$; (b) $k \cong 1$; (c) $k \gg 1$.

Figura 3.7: Função densidade de probabilidade da distribuição m-Nakagami. 62 
Figura 4.1: Percurso realizado ao longo das medições.

Figura 4.2: Bloco de transmissão do sistema de medidas.

Figura 4.3: Bloco de recepção do sistema de medidas.

Figura 4.4: Reta de amplificação do equipamento Milmega.

Figura 4.5: Antena painel, modelo HG3515P-120.

Figura 4.6: Diagrama de irradiação da antena painel modelo HG3515P-120: (a) plano horizontal; (b) plano vertical.

Figura 4.7: Antena omni direcional, modelo HG3505RD-RSP.

Figura 4.8: Diagrama de irradiação da antena omni direcional (a) plano horizontal (b) plano vertical.

Figura 4.9: Fotografia dos blocos de transmissão e recepção em ambiente laboratorial.

Figura 4.10: Varredura do espectro entre $3,4 \mathrm{GHz}$ a $3,6 \mathrm{GHz}$.

Figura 4.11: Região abrangida pela terceira campanha de medição.

Figura 4.12: Sinal OFDM, no domínio da frequência, transmitido ao longo das medições.

Figura 4.13: Disposição das subportadoras do sinal transmitido (domínio da frequência).

Figura 4.14: Pontos de referência de parâmetros do balanço de potência.

Figura 5.1: Distribuição cumulativa das subportadoras piloto de um setor da Rua Mário Ribeiro, a $315 \mathrm{~m}$ da antena transmissora. Ambiente LOS.

Figura 5.2: Distribuição cumulativa das subportadoras piloto de um setor da Rua Mário Ribeiro, a 1,5 km da antena transmissora. Ambiente LOS.

Figura 5.3: Distribuição cumulativa das subportadoras piloto de um setor da Rua Padre Leonel. Ambiente NLOS.

Figura 5.4: Distribuição cumulativa das subportadoras piloto de um setor da Rua Marquês de São Vicente. Ambiente NLOS.

Figura 5.5: Função distribuição cumulativa do nível de sinal obtido em um setor da Rua Mário Ribeiro (LOS) e distribuição cumulativa teórica Rayleigh e de m-Nakagami.

Figura 5.6: Função distribuição cumulativa do nível de sinal obtido em um setor da Rua Padre Leonel (NLOS) e distribuição cumulativa teórica Rayleigh e de m-Nakagami. 
Figura 5.7: Comparação de sinais obtidos em LOS e NLOS.

Figura 5.8: Sinal recebido em ambiente de LOS.

Figura 5.9: Taxa de cruzamento de nível e duração média do desvanecimento da primeira subportadora piloto de um setor em situação de LOS.

Figura 5.10: Taxa de cruzamento de nível e duração média do desvanecimento da segunda subportadora piloto de um setor em situação de LOS.

Figura 5.11: Taxa de cruzamento de nível e duração média do desvanecimento da terceira subportadora piloto de um setor em situação de LOS.

Figura 5.12: Taxa de cruzamento de nível e duração média do desvanecimento da quarta subportadora piloto de um setor em situação de LOS.

Figura 5.13: Taxa de cruzamento de nível e duração média do desvanecimento da quinta subportadora piloto de um setor em situação de LOS.

Figura 5.14: Taxa de cruzamento de nível e duração média do desvanecimento da sexta subportadora piloto de um setor em situação de LOS.

Figura 5.15: Taxa de cruzamento de nível e duração média do desvanecimento da sétima subportadora piloto de um setor em situação de LOS.

Figura 5.16: Taxa de cruzamento de nível e duração média do desvanecimento da oitava subportadora piloto de um setor em situação de LOS.

Figura 5.17: Taxa de cruzamento de nível e duração média do desvanecimento da primeira subportadora piloto de um setor em situação de NLOS.

Figura 5.18: Taxa de cruzamento de nível e duração média do desvanecimento da segunda subportadora piloto de um setor em situação de NLOS.

Figura 5.19: Taxa de cruzamento de nível e duração média do desvanecimento da terceira subportadora piloto de um setor em situação de NLOS.

Figura 5.20: Taxa de cruzamento de nível e duração média do 
desvanecimento da quarta subportadora piloto de um setor em situação de NLOS.

Figura 5.21: Taxa de cruzamento de nível e duração média do desvanecimento da quinta subportadora piloto de um setor em situação de NLOS.

Figura 5.22: Taxa de cruzamento de nível e duração média do desvanecimento da sexta subportadora piloto de um setor em situação de NLOS.

Figura 5.23: Taxa de cruzamento de nível e duração média do desvanecimento da sétima subportadora piloto de um setor em situação de NLOS.

Figura 5.24: Taxa de cruzamento de nível e duração média do desvanecimento da oitava subportadora piloto de um setor em situação de NLOS.

Figura 5.25: Correlação da quadragésima subportadora (primeira subportadora piloto) de um setor, em LOS, da Rua Mário Ribeiro.

Figura 5.26: Correlação da 166 ${ }^{\text {a }}$ subportadora (sexta subportadora piloto) de um setor, em LOS, da Rua Mário Ribeiro.

Figura 5.27: Correlação da quadragésima subportadora (primeira subportadora piloto) de um setor, em LOS, da Rua Vice-Governador Rubens Berardo.

Figura 5.28: Correlação da quadragésima subportadora (primeira subportadora piloto) de um setor, em LOS, da Rua Padre Leonel.

Figura 5.29: Correlação das subportadoras piloto de um setor, em LOS, da rua Mário Ribeiro.

Figura 5.30: Correlação das subportadoras piloto de um setor, em NLOS, da Rua Vice-Governador Rubens Berardo. 


\section{Lista de tabelas}

Tabela 1.1: Classificação de acesso fixo a móvel de acordo com o padrão WiMAX.

Tabela 1.2: Profile criado pelo WiMAX fórum para o padrão IEEE 802.16-2004.

Tabela 1.3: Parâmetros do símbolo OFDM-256

Tabela 1.4: Profile para o padrão IEEE 802.16e-2005

24

Tabela 1.5: Parâmetros de um símbolo OFDM e OFDMA.

Tabela 5.1: Médias da banda de coerência em ambientes LOS e NLOS.

Tabela 5.2: Valor calculado de delay spread, com base na média ponderada da BC, para ambientes LOS e NLOS.

Tabela 5.3: Valor calculado de delay spread, com base na média aritmética da BC, para ambientes LOS e NLOS.

Tabela 5.4: Valores de delay spread calculados a partir da regressão linear sugerida por [10]. 